\title{
Les cancers de la cavité buccale : affection à prédominance féminine à Ouagadougou
}

\author{
Augustin Tozoula Bambara ${ }^{1,}{ }^{*}$, Mathieu Millogo ${ }^{2}$, Tarcissus Konsem ${ }^{2}$, \\ Hierrhum Aboubacar Bambara ${ }^{1}$, Dieudonné Ouédraogo ${ }^{2}$, Si Simon Traoré ${ }^{1}$ \\ ${ }^{1}$ Service de chirurgie générale et digestive, CHU Yalgado Ouédraogo (Ouagadougou) \\ ${ }^{2}$ Service de stomatologie et chirurgie maxillo-faciale, CHU Yalgado Ouédraogo (Ouagadougou)
}

(Reçu le 27 janvier 2015, accepté le 9 mars 2015)

Mots clés : cancer / cavité buccale

Key words: cancer / oral cavity
Résumé - Objectif : Nous avons mené une étude rétrospective dans le but de présenter les caractéristiques des cancers de la cavité buccale à travers une comparaison entre les deux sexes au CHU Yalgado Ouédraogo (Ouagadougou, Burkina Faso). Patients et méthodes : Il s'agit d'une étude rétrospective à partir de l'examen des dossiers de 79 patients reçus en consultation entre janvier 2003 et décembre 2014. Tous les patients avec un diagnostic histologique d'une tumeur maligne intra-orale ont été inclus. Les caractéristiques démographiques des patients (âge, sexe) et les principales caractéristiques des tumeurs malignes (localisation, type histologique) ont été explorées. Les variables ont été comparées selon le sexe à l'aide du test T de Student. Résultats : Nous avons colligé 79 patients âgés en moyenne de 56 ans. La prédominance féminine était nette (sex ratio $=0,55)$. Les femmes étaient à $90,2 \%$ sans emploi tandis que la proportion d'hommes sans emploi s'élevait à $60,7 \%$ $(p=0,004)$. Les hommes étaient tous fumeurs alors que la majorité des femmes chiquaient le tabac. Chez les sujets masculins, les cancers de la langue étaient les plus fréquents $(32,1 \%)$, suivis des cancers du palais et des cancers de la joue. Chez les femmes, les localisations les plus fréquentes étaient les lèvres (37,3\%), la joue et la gencive. Les hommes ont consulté à des stades plus avancés que les femmes. Le carcinome épidermoïde était prédominant dans les deux sexes. Conclusion : Le bas niveau socio-économique joue probablement un rôle important dans cette prédominance féminine.

\begin{abstract}
Cancers of the oral cavity: a female-predominant incidence in Ouagadougou. Oral cavity cancers affect men more often than women. A female predominance has been observed in consultation at Yalgado Ouedraogo Teaching Hospital. Objective: We conducted a retrospective study with the aim of showing the characteristics of these cancers through a comparison between the two sexes at Yalgado Ouedraogo Teaching Hospital (Ouagadougou, Burkina Faso). Materials and Methods: A retrospective study from 79 medical files of patients consulting between January 2013 and December 2014 was conducted. All of them were affected by a histological confirmed cancer of the oral cavity. The demographic characteristics of the patients (age and gender) and main characteristics of the malign tumor (localization, histological type) were investigated. Parameters were compared according to gender with the Student t-test. Results: We collected 79 patients with an average age of 56 (standard deviation: 15 years). The female predominance was clear (sex ratio $=0.55)$ and related to all the age groups. The proportion of patients who had no job was about $90.2 \%$ for women versus $60.7 \%$ for men $(p=0.004)$. The men were all smokers, while some women chewed tobacco. Tongue cancer was more frequent in the male subject $(32.1 \%)$, followed by palate cancer and cheek cancer. Among women, the most frequent localizations were the lips $(37.3 \%)$, the cheeks and the gum. Men consulted at more advanced stages than women. Epidermoid carcinoma was the prevalent histological type in both sexes. Conclusion: Jointly with the classical risk factors, alcohol and tobacco addictions, the low socio-economic level plays a significant role in this female prevalence.
\end{abstract}

\footnotetext{
* Correspondance : tozoula@yahoo.fr
} 


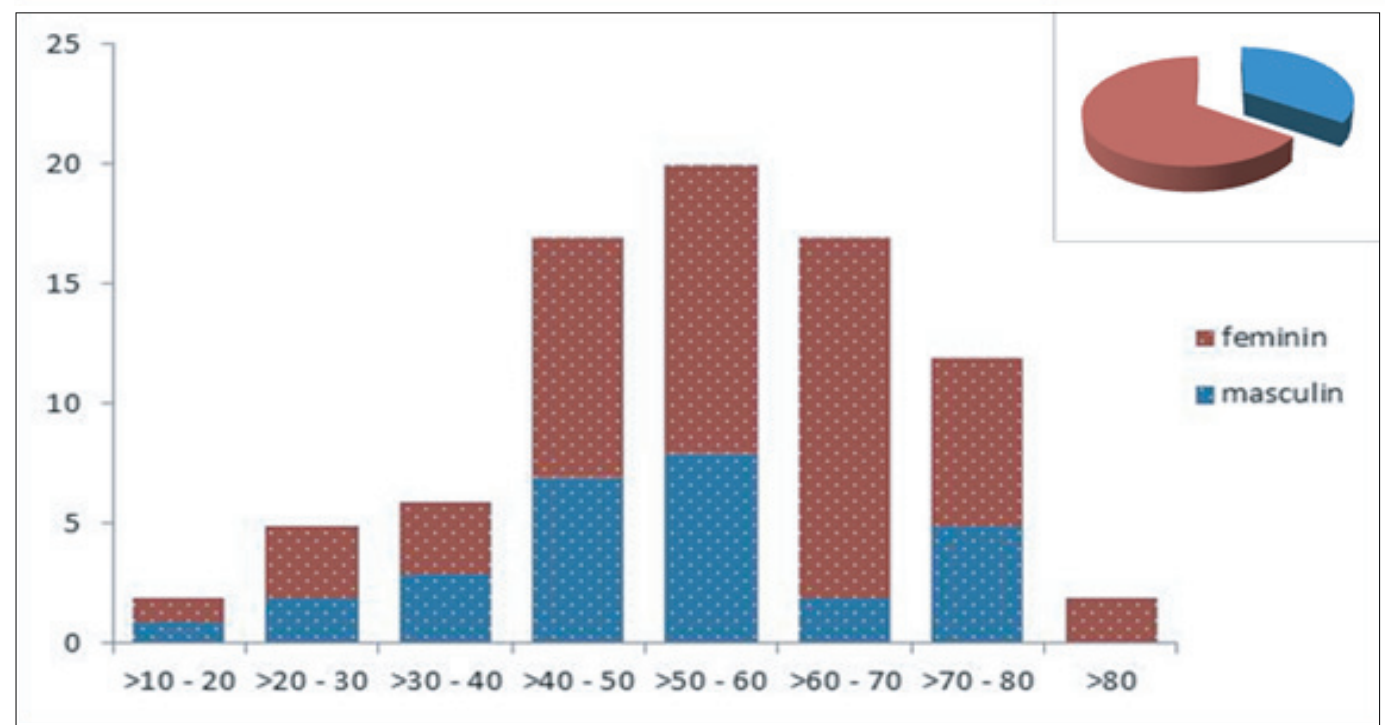

Fig. 1. Répartition des patients selon les tranches d'âge et le sexe.

Fig. 1. Distribution of patients according to age groups and gender.

\section{Introduction}

Les cancers de la cavité buccale sont des affections graves au double plan fonctionnel et vital. En effet, ils ont une importante répercussion sur la mastication, la déglutition et la phonation [1]. Les variations de sa répartition sont en rapport avec la consommation d'alcool et de tabac qui constituent les principaux facteurs de risque. Ces habitudes étant majoritairement masculines, la prédominance masculine des cancers de la cavité buccale fait quasiment l'unanimité dans la littérature $[2,3]$. Une prédominance féminine a été observée en consultation de stomatologie et d'oncologie au CHU Yalgado Ouédraogo, suggérant le rôle important joué par d'autres facteurs de risque que le tabagisme et l'alcoolisme. Nous avons donc mené cette étude dans le but de présenter les caractéristiques épidémiologiques et cliniques de ces cancers à travers une comparaison entre les deux sexes.

\section{Patients et méthodes}

Il s'est agi d'une étude rétrospective menée sur la base de dossiers médicaux des patients reçus en consultation de stomatologie et d'oncologie au CHU Yalgado Ouédraogo du $1^{\text {er }}$ janvier 2003 au 31 décembre 2014. Ont été inclus dans l'étude les patients ayant présenté un cancer histologiquement confirmé, développé aux dépens d'un constituant de la cavité buccale. La cavité buccale a été définie comme la première portion du tube digestif comprise entre la voute palatine en haut, le plancher buccal en bas, les lèvres en avant et la luette en arrière. Elle est complétée par le vestibule buccal qui est un espace en fer à cheval compris entre les arcades dentaires, les joues et les lèvres. Les cancers du versant cutané des lèvres non pas été inclus. Nous avons analysé les potentiels facteurs de risque, les aspects cliniques, paracliniques et les traitements reçus. Pour chaque tumeur, l'extension a été appréciée et une classification TNM (UICC 2010) a été établie [4]. Les variables étudiées ont été comparées selon le sexe. Les proportions ont été comparées à l'aide du test du $\mathrm{Chi}^{2}$ ou de Fisher exact ; le test de Student a servi à la comparaison des moyennes. Un seuil de significativité de 0,05 a été retenu pour toutes ces analyses.

\section{Résultats}

Le service de chirurgie maxillo-faciale et stomatologie du CHU Yalgado Ouédraogo reçoit en moyenne 441 consultations pour affections non traumatiques par an. Les femmes représentent $50,6 \%$ de ces consultations. Nous avons colligé 79 patients qui étaient âgés en moyenne de 56 ans (écart type : 15 ans), soit une moyenne de 6,6 cas par an. La prédominance féminine était nette $($ sex ratio $=0,55)$ et a concerné toutes les tranches d'âge (Fig. 1). Le délai moyen de consultation a été de 18 mois dans les deux sexes. Les femmes étaient à $90,2 \%$ sans emploi tandis que la proportion $d^{\prime}$ hommes sans emploi s'élevait à 60,7\% ( $\left.p=0,004\right)$ (Tab. I). Une proportion similaire de patients des deux sexes était des consommateurs de tabac. Les hommes étaient tous des fumeurs, pendant que les femmes chiquaient le tabac (Tab. I). Chez les sujets de sexe masculin, les cancers de la langue étaient les plus fréquents $(32,1 \%)$, suivis des cancers du palais $(25 \%)$ et des cancers de la joue $(21,4 \%)$. D'autres localisations étaient plus rarement enregistrées (Fig. 2 et 3 ). 
Tableau I. Statut professionnel, résidence et addictions des patients.

Table I. Employment status, residence and addiction in patients.

\begin{tabular}{|c|c|c|c|c|c|}
\hline & \multicolumn{2}{|c|}{ Féminin $(n=51)$} & \multicolumn{2}{|c|}{ Masculin $(n=28)$} & \multirow{2}{*}{ Valeur $p$} \\
\hline & Effectifs & $\%$ & Effectifs & $\%$ & \\
\hline Sans emploi & 46 & 90,2 & 17 & 60,7 & 0,004 \\
\hline Résidence rurale & 30 & 58,8 & 12 & 42,8 & 0,2 \\
\hline \multicolumn{6}{|l|}{ Addiction } \\
\hline - Tabac & 12 & 23,5 & 6 & 21,4 & \\
\hline - Alcool & 4 & 7,8 & 3 & 10,7 & \\
\hline - Alcool et tabac & 2 & 3,9 & 4 & 14,3 & $0,1^{*}$ \\
\hline - Aucune & 23 & 45,1 & 7 & 25,0 & \\
\hline - Non précisée & 10 & 19,6 & 8 & 28,6 & \\
\hline
\end{tabular}

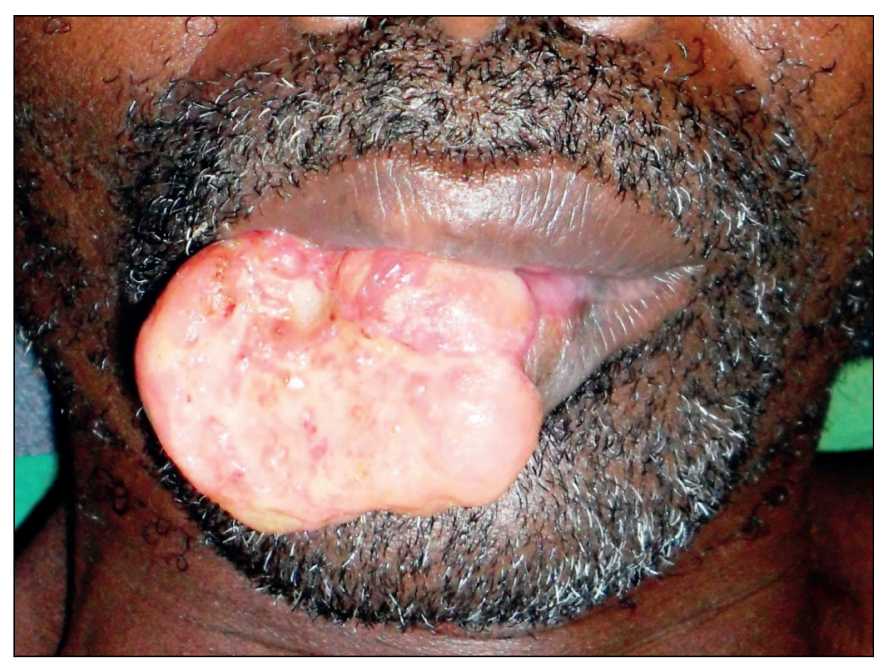

Fig. 2. Carcinome indifférencié de la lèvre inférieure chez un patient de 43 ans.

Fig. 2. Undifferentiated carcinoma of the lower lip in a 43 years old male patient.

Chez les femmes, les localisations les plus fréquentes étaient la lèvre $(37,3 \%)$, la joue $(19,6 \%)$ et la gencive $(13,7 \%)$ (Tab. II). Là encore, d'autres localisations étaient enregistrées (Fig. 4). Les hommes ont consulté à des stades plus avancés que les femmes. En effet, la proportion de stades inférieurs au stade T4 était de $63,3 \%$ chez les femmes, contre $48,1 \%$ chez les hommes (Tab. III). Le carcinome épidermoïde était le type histologique prédominant dans les deux sexes. Le carcinome adénoïde kystique occupait le second rang chez les femmes (Tab. IV). Quatorze patients ont bénéficié d'une chirurgie d'exérèse tumorale. Aucun patient n'a bénéficié de radiothérapie. Une chimiothérapie palliative a été administrée à 8 patients.

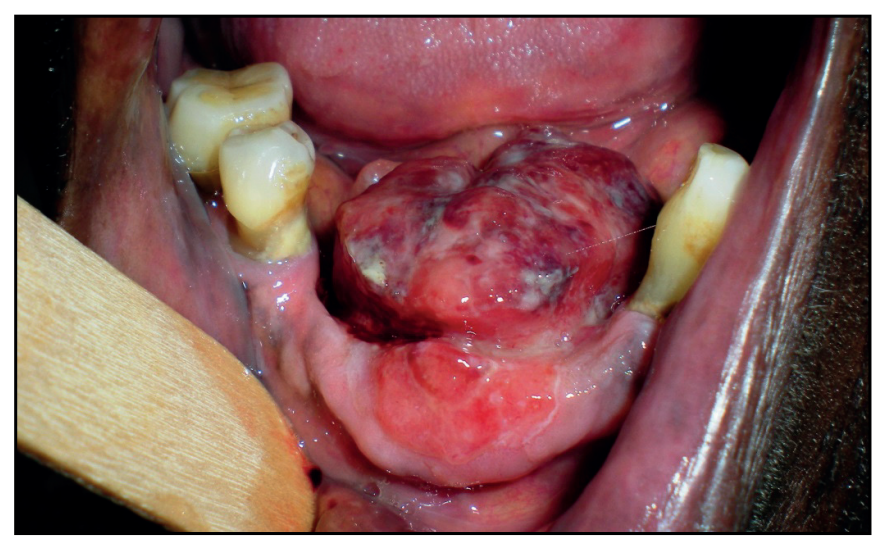

Fig. 3. Carcinome adénoïde kystique du plancher buccal chez un patient de 46 ans.

Fig. 3. Adenoid cystic carcinoma of the mouth floor in a 46 years old female patient.

\section{Discussion}

Les cancers de la cavité buccale sont relativement fréquents. En 2008, 263900 nouveaux cas ont été diagnostiqués dans le monde, avec un taux d'incidence standardisé à l'âge de 2,5 pour 100000 hommes, contre 2,1 pour 100000 femmes par an en Afrique de l'Ouest [5]. Au Burkina Faso, ils représentent le $6^{\mathrm{e}}$ cancer histologiquement confirmé après les cancers du sein, du col utérin, de la peau, des ganglions et de l'estomac, avec une fréquence annuelle de 12 cas [6]. Notre étude a révélé une fréquence annuelle de 6,6 cas au CHU Yalgado Ouédraogo qui est le centre de référence du pays. Ce centre reçoit donc la majorité des cas, d'autant plus qu'il se situe dans la capitale, lieu de concentration des trois laboratoires d'anatomie pathologique en activité dans le pays durant la période concernée. 
Tableau II. Les localisations tumorales selon le sexe.

Table II. The tumor sites by sex.

\begin{tabular}{|c|c|c|c|c|c|}
\hline \multirow{2}{*}{ Localisations } & \multicolumn{2}{|c|}{ Féminin } & \multicolumn{2}{|c|}{ Masculin } & \multirow{2}{*}{ Valeur $\mathrm{p}$} \\
\hline & Effectifs & $\%$ & Effectifs & $\%$ & \\
\hline Lèvres & 19 & 37,3 & 3 & 10,7 & \multirow{7}{*}{$0,02^{*}$} \\
\hline Joues & 10 & 19,6 & 6 & 21,4 & \\
\hline Gencives & 7 & 13,7 & 3 & 10,7 & \\
\hline Palais & 6 & 11,8 & 7 & 25,0 & \\
\hline Langue & 5 & 9,8 & 9 & 32,1 & \\
\hline Plancher & 4 & 7,8 & 0 & 0,0 & \\
\hline Total & 51 & 100,0 & 28 & 100,0 & \\
\hline
\end{tabular}

*Test exact de Fisher

(Fisher's exact test)

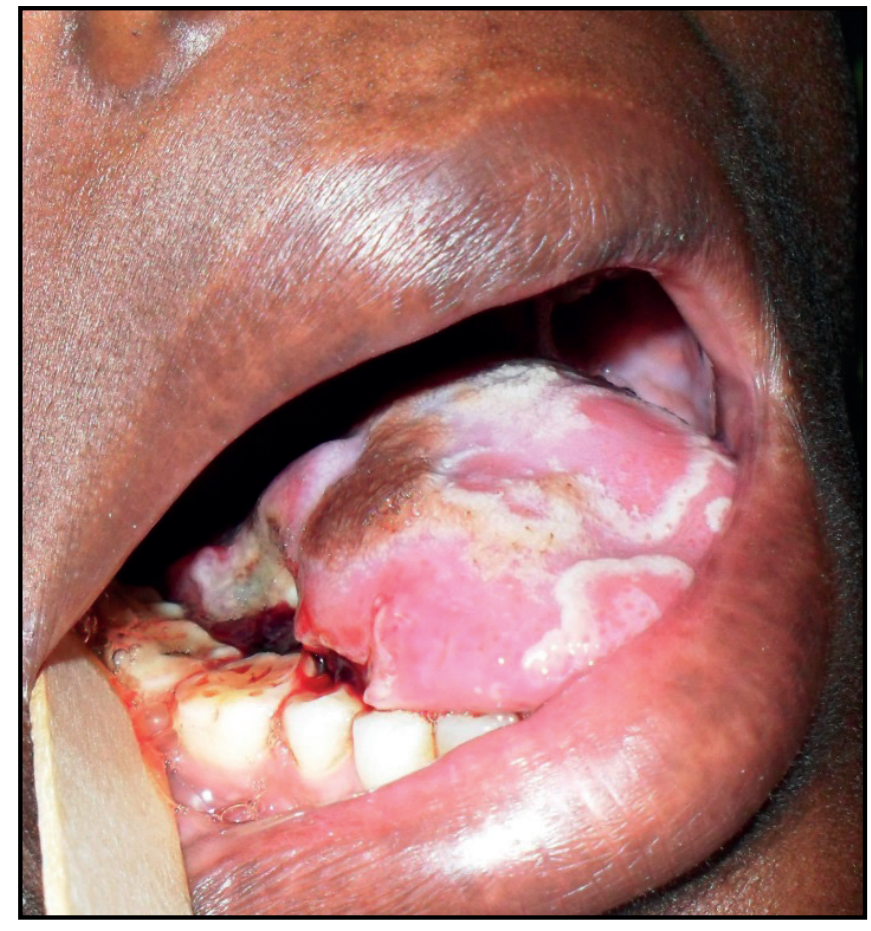

Fig. 4. Carcinome épidermoïde de la langue chez une patiente de 31 ans.

Fig. 4. Squamous cell carcinoma of the tongue in a 31 years old female patient.

La prédominance féminine a été nette dans notre étude, estimée à 2 femmes pour un homme. Cette prédominance diffère considérablement des données de la littérature qui font quasiment l'unanimité sur la prédominance masculine de cette maladie [5, 3]. Kayembé a toutefois trouvé une prédominance féminine dans des proportions similaires en République démocratique du Congo [7]. Nous avons donc exploré certaines hypothèses susceptibles $d$ 'expliquer cette prédominance féminine dans le contexte de la présente étude.
Tableau III. Répartition des patients selon la classification TNM au diagnostic (sarcomes exclus).

Table III. Distribution of patients according to the TNM classification at diagnosis (excluded sarcomas).

\begin{tabular}{|c|c|c|c|c|}
\hline \multirow{2}{*}{ TNM } & \multicolumn{2}{|c|}{ Féminin } & \multicolumn{2}{|c|}{ Masculin } \\
\hline & Effectifs & $\%$ cum. & Effectifs & $\%$ cum. \\
\hline T2 NO MO & 5 & 10,2 & 2 & 7,4 \\
\hline T2 N1 MO & 1 & 12,2 & 0 & 7,4 \\
\hline T2 N2a MO & 1 & 14,3 & 0 & 7,4 \\
\hline T3 NO MO & 16 & 46,9 & 8 & 37,0 \\
\hline T3 N1 M0 & 1 & 49,0 & 2 & 44,4 \\
\hline T3 N2b MO & 1 & 51,0 & 0 & 44,4 \\
\hline T3 N2C MO & 6 & 63,3 & 1 & 48,1 \\
\hline T4 NO MO & 7 & 77,6 & 4 & 63,0 \\
\hline T4 N1 M0 & 1 & 79,6 & 0 & 63,0 \\
\hline T4 N2a MO & 1 & 81,6 & 1 & 66,7 \\
\hline T4 N2b MO & 3 & 87,8 & 0 & 66,7 \\
\hline T4 N2b M1 & 2 & 91,8 & 1 & 70,4 \\
\hline T4 N2C MO & 3 & 98,0 & 5 & 88,9 \\
\hline T4 N2c M1 & 0 & 98,0 & 1 & 92,6 \\
\hline Tx N1 M0 & 1 & 100,0 & 0 & 92,6 \\
\hline Tx N2b M0 & 0 & 100,0 & 1 & 96,3 \\
\hline Tx N3 M1 & 0 & 100,0 & 1 & 100,0 \\
\hline TOTAL & 49 & 100,0 & 27 & 100,0 \\
\hline
\end{tabular}

$\%$ cum. : fréquence cumulée

(cumulative frequency)

Le service de chirurgie maxillo-faciale et de stomatologie reçoit en consultation autant d'hommes que de femmes pour des affections non traumatiques. La prédominance féminine des cancers de la cavité buccale n'est donc pas liée à une plus grande fréquentation des services de santé par les femmes. Dans une étude menée par Varenne sur les raisons de la fréquentation des services de soins bucco-dentaires dans l'aire géographique de Ouagadougou, 51,8 \% des demandeurs de 
Tableau IV. Répartition des types histologiques selon le sexe.

Table IV. Distribution of histological types by sex.

\begin{tabular}{|c|c|c|c|c|c|}
\hline \multirow{2}{*}{ Histologie } & \multicolumn{2}{|c|}{ Féminin } & \multicolumn{2}{|c|}{ Masculin } & \multirow{2}{*}{ Valeur $\mathrm{p}$} \\
\hline & Effectifs & $\%$ & Effectifs & $\%$ & \\
\hline C. épidermoïde & 41 & 77,4 & 25 & 89,3 & \multirow{6}{*}{$0,30^{*}$} \\
\hline C. adénoïde kystique & 5 & 9,4 & 0 & 0,0 & \\
\hline Sarcome & 3 & 5,7 & 1 & 3,6 & \\
\hline Adénocarcinome & 2 & 3,8 & 1 & 3,6 & \\
\hline C. indifférencié & 0 & 0,0 & 1 & 3,6 & \\
\hline Total & 51 & 100,0 & 28 & 100,0 & \\
\hline
\end{tabular}

*Test exact de Fisher

(Fisher's exact test)

C. : Carcinome

(carcinoma)

soins bucco-dentaires étaient des femmes [8]. Cette proportion ne diffère pas significativement de celle des hommes puisque les femmes représentaient $51,7 \%$ de la population générale du Burkina Faso au dernier recensement général de la population [9].

La consommation de tabac et d'alcool constituent les principaux facteurs de risque des cancers de la cavité buccale, si bien que les variations de la répartition de cette maladie sont liées à ces habitudes. Dans notre étude, les hommes et les femmes consommaient le tabac dans des proportions similaires, mais le mode de consommation était différent. En effet, les hommes étaient fumeurs de tabac, tandis que les femmes le chiquaient. Cette habitude consistant à placer une portion de tabac entre la lèvre inférieure et les dents pour en extraire le jus tout en rejetant le surplus a été, elle aussi, associée aux cancers de la cavité buccale [10]. Les dossiers cliniques des patients ne mentionnaient cependant pas les quantités de tabac et d'alcool consommées ainsi que les durées d'exposition. Cela ne nous permet pas d'affirmer que les femmes de cette série sont plus exposées au tabac que les hommes.

Outre la consommation d'alcool et de tabac, l'infection au virus du papillome humain (HPV), virus infectant couramment la sphère génitale féminine, a été associée au cancer de la cavité buccale avec un risque relatif de l'ordre de 1,5 [11]. Aucune étude épidémiologique n'a évalué la prévalence de cette infection dans la population burkinabé. Nous pouvons cependant minimiser le rôle de ce virus dans la prédominance féminine des cancers de la cavité buccale puisqu'aucune corrélation entre l'infection génitale au HPV et la colonisation buccale par le même virus n'a encore été démontrée [12].

De nombreuses études ont récemment mis en évidence le rôle direct de la mauvaise hygiène buccodentaire dans la survenue des cancers de la cavité buccale [13]. Nous n'avons pas pu évaluer l'hygiène bucco-dentaire des patients de notre étude. Nous pouvons néanmoins nous référer à l'étude menée par Varenne qui a évalué l'état de santé bucco-dentaire en milieu rural et urbain au Burkina Faso [14]. Cette étude a révélé que la moyenne de dents cariées, dents absentes et dents obturées était de 6,1 entre 35 et 44 ans, avec des chiffres plus élevés chez les femmes que chez les hommes (8 vs. 4,$4 ; p<0,001$ ). L'état parodontal était plus altéré chez les femmes et chez les personnes vivant en milieu rural. La mauvaise hygiène bucco-dentaire pourrait donc avoir joué un rôle important dans la survenue de cancer de la cavité buccale chez les femmes de cette étude.

Dans notre étude, les localisations tumorales étaient différentes selon le sexe. Les cancers de la langue et du palais prédominaient chez l'homme, tandis que les cancers de la lèvre et de la joue étaient les plus fréquents chez la femme. La langue est la localisation tumorale buccale la plus fréquente dans de nombreux pays tous sexes confondus [15]. Son incidence est en augmentation dans les pays occidentaux, particulièrement chez les sujets jeunes de sexe féminin [16]. L'infection au HPV en serait la principale cause [5]. Les cancers de la langue restent néanmoins les cancers buccaux les plus fréquents chez l'homme du fait de la part de risque attribuable au tabac et à l'alcool, habitudes majoritairement masculines. La répartition selon le sexe des cancers de la lèvre, de la joue et de la gencive est mal connue car ils sont souvent étudiés simultanément avec les autres cancers des voies aérodigestives supérieures. Le tabagisme, quel que soit le mode, est là aussi le premier facteur de risque identifié. La survenue de ces cancers est plus fortement liée au fait de chiquer ou de mâcher le tabac (mode de consommation des femmes de notre série) qu'au fait de le fumer [10]. Cela pourrait expliquer la prédominance des cancers labiaux, jugaux et gingivaux chez les sujets de sexe féminin dans cette étude.

Plus de la moitié des cancers de la cavité buccale sont diagnostiqués à un stade III ou IV [17]. Le bas niveau socio-économique qui compromet l'accès aux soins bucco-dentaires a souvent été incriminé [17]. Les consultations régulières et 
systématiques chez le dentiste permettent, en effet, la détection des lésions précancéreuses de la cavité buccale ainsi que le diagnostic précoce des cancers buccaux [18]. Dans notre étude, les stades avancés T3 et T4 représentent $84,8 \%$. Cette proportion très élevée pourrait s'expliquer par le long délai de consultation (18 mois en moyenne), en partie lié à la faible fréquentation des services de soins bucco-dentaires. Comme l'a montré Varenne dans une étude menée dans les centres de soins bucco-dentaires à Ouagadougou, la majorité des patients ne consultaient que devant un problème bucco-dentaire (caries dans 52,4\% et douleur dans $60 \%$ des cas) et seuls $15,9 \%$ étaient des ménagères [8].

Les carcinomes épidermoïdes étaient largement majoritaires dans les deux sexes. Nous avons néanmoins observé plus de variétés histologiques chez les femmes que chez les hommes.

En l'absence d'une couverture maladie universelle au Burkina Faso, le coût des traitements antitumoraux est entièrement à la charge des patients. Les coûts élevés des médicaments anticancéreux ainsi que la non-disponibilité de la radiothérapie à Ouagadougou ont rendu leur prise en charge souvent inadéquate.

\section{Conclusion}

Les cancers de la cavité buccale intéressent beaucoup plus les femmes dans notre contexte. Conjointement aux facteurs de risque classiques que sont l'alcoolisme et le tabagisme, le bas niveau socio-économique joue un rôle important dans cette prédominance féminine. La différence des modes de tabagisme expliquerait les localisations tumorales. Le retard à la consultation, les insuffisances du plateau technique et l'état de pauvreté des patients compromettent leur survie.

Conflits d'intérêt : aucun.

\section{Références}

1. Daly-Schveitzer N. Cancérologie clinique, $3^{\mathrm{e}}$ ed. Masson, Paris, 2008.

2. Warnakulasuriya S. Global epidemiology of oral and oropharyngeal cancer. Oral Oncol 2009;45:309-16.

3. de Camargo Cancela M, Voti L, Guerra-Yi M, Chapuis F, Mazuir M, Curado MP. Oral cavity cancer in developed and developing countries: population-based incidence. Head Neck 2010;32:357-67.
4. International Union against Cancer (UICC). TNM Classification of Malignant Tumours. $7^{\text {th }}$ ed. Sobin LH, Gospodarowicz MK, Wittekind CH, Eds. John Wiley and Sons, New York, 2011.

5. Jemal A, Bray F, Center MM, Frelay J, Ward E, Forman D. Global Cancer Statistics. CA Cancer J Clin 2011;61:69-90.

6. Lompo-Goumbri OM, Domagni OE, Sanou AM, Konsegré V, Soudré RB. Aspects épidémiologiques et histopathologiques des cancers au Burkina Faso. Journal africain du cancer 2009;1:207-11.

7. Kayembé MK, Kalengayi MM. Histological and epidemiological profile of oral cancer in Congo (Zaïre). Odontostomatol Trop 1999;88:29-32.

8. Varenne B, Msellati P, Zoungrana C, Fournet F, Salem G. Reasons for attending dental-care services in Ouagadougou, Burkina Faso. Bull World Health Organ 2005;83:650-55.

9. INSD, MEF, Burkina Faso. Recensement général de la population et de l'habitat, 2006 (General Population Census, 2006). MEF/ INSD: Ouagadougou, Burkina Faso, 2009.

10. Jayalekshmi PA, Gangadharan P, Akiba S, Nair RRK, Tsuji M, Rajan B. Tobacco chewing and female oral cavity cancer risk in Karunagappally cohort, India. Br J Cancer 2009;100:848-52.

11. Herrero R, Castellsagué X, Pawlita M, Lissowska J, Kee F, Balaram $P$ et al. Human papillomavirus and oral cancer: the international agency for research on cancer multicenter study. J Natl Cancer Inst 2003;95(23):1772-83.

12. Meyera MF, Huebbers CU, Siefer OG, Vent J, Engbert I, Eslick GD et al. Prevalence and risk factors for oral human papillomavirus infection in 129 women screened for cervical HPV infection. Oral Oncol 2014;50:27-31.

13. Akram S, Mirza T, Mirza MA, Qureshi M. Emerging patterns in clinico-pathological spectrum of oral cancers. Pak J Med Sci 2013;29(3):783-7.

14. Varenne B, Petersen PE, Ouattara S. oral health status of children and adults in urban and rural areas of Burkina Faso, Africa. Int Dent J 2004;54:83-9.

15. Sutandyo N, Ramli R, Sari L, Soeis DS. Profile and survival of tongue cancer patients in « Dharmais » Cancer Hospital, Jakarta. Asian Pac J Cancer Prev 2014;15(5):1971-5.

16. Reddy V, Cundall-Curry D, Bridger MWM. Trends in the incidence rates of tonsil and base of tongue cancer in England, 1985-2006. Ann R Coll Surg Engl 2010;92(8):655-9.

17. Adrien J, Bertolus C, Gambotti L, Mallet A, Baujat B. Why are head and neck squamous cell carcinoma diagnosed so late? Influence of health care disparities and socio-economic factors. Oral Oncol 2014;50(2):90-7.

18. Poh CF, Williams PM, Zhang L, Rosin MP. Attention ! Appel aux dentistes pour le dépistage du cancer de la bouche. Journal of Canadian Dental Association 2006;72(5):413-6. 